\title{
EFFECTS OF FASTING AND GENDER ON OCHRATOXIN A TOXICOKINETICS IN F344 RATS
}

A. Vettorazzi ${ }^{a}$, I.F.Trocóniz ${ }^{b}$, E. Gonzalez-Peñas ${ }^{c}$, L.A. Corcuera ${ }^{a}$, L. Arbillaga ${ }^{a}$, A.G. Gil ${ }^{a}$, J.M. Nagy ${ }^{d}$, P.G. Mantle ${ }^{\mathrm{e}}$, A. López de Cerain ${ }^{\mathrm{a}^{*}}$.

${ }^{a}$ Department of Nutrition and Food Sciences, Physiology and Toxicology, Faculty of

Pharmacy, University of Navarra, Irunlarrea 1, E-31008. Pamplona, Spain.

.$^{b}$ Department of Pharmacy and Pharmaceutical Technology, Faculty of Pharmacy, University of Navarra, Irunlarrea 1, E-31008. Pamplona, Spain

${ }^{\circ}$ Department of Organic and Pharmaceutical Chemistry, Faculty of Pharmacy, University of Navarra, Irunlarrea 1, E-31008. Pamplona, Spain

${ }^{d}$ Institute of Biomedical Engineering, Imperial College London, London SW7 2AZ, UK

${ }^{\text {e}}$ Center for Environmental Policy, Imperial College London, London SW7 2AZ, UK

\section{Address correspondence to:}

Adela López de Cerain, Ph.D.

Department of Nutrition and Food Sciences, Physiology and Toxicology,

Faculty of Pharmacy, University of Navarra

Irunlarrea 1, E-31008. Pamplona, Spain

Tel.: +34 948425653

Fax: +34948425652

Email: acerain@unav.es

Running title: Effects of food and gender on OTA toxicokinetics

Keywords: ochratoxin A, toxicokinetic, bioavailability, male and females F344 rat, food, alpha-2u-globulin

\section{Abbreviations:}

a2u: alpha-2u-globulin. -2LL: -2xlog (likelihood). BEN: Balkan endemic nephropathy. b.w.: body weight. $\mathrm{CMAX}_{\text {obs: }}$ maximum observed concentration. CV(\%): coefficient of variation. CL: Total plasma clearance. F: relative biovailability. F344: Fisher 344 rats. HPLC-FLD: high pressure liquid chromatography equipped with a fluorescence detector. IAV: inter-animal variability. $\mathbf{K}_{\mathbf{A}}$ : first order rate constant of absorption. LOD: limit of detection. LOQ: limit of quantification. MWF: micronized wheat fibers. OTA: ochratoxin A. Q: distribution clearance.

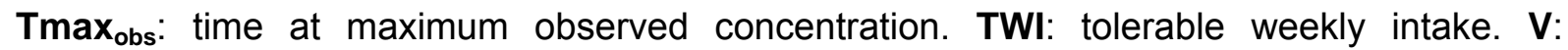
apparent volume of distribution of the central compartment. $\mathbf{V}_{\mathbf{T}}$ : apparent volume of distribution of the peripheral compartment. RSD: relative standard deviation. SDS-PAGE: sodium dodecyl sulfate polyacrylamide gel electrophoresis. WGT: weight. 


\section{Abstract}

Ochratoxin A (OTA) is a mycotoxin that causes renal tumors in rats, particularly in males. The mechanism below the marked sex-difference sensitivities to OTA is still unknown. In previous studies performed in fed conditions (Vettorazzi et al. 2008) mature males presented a different kinetic profile than young and female rats. The objective of the present study was to evaluate the effect of fasting and the involvement of the male-specific protein alpha-2uglobulin on OTA kinetics in mature F344 rats of both sexes. A $24 \mathrm{~h}$ kinetic study in fasted conditions has been performed and compared with fed conditions. Food ingestion has been controlled during two months and the presence of alpha-2u-globulin in the urine has been analyzed with SDS-gradient mini-gel electrophoresis.

Fasting tends to increase the maximum plasma concentrations and the rate of absorption. The relative bioavailability is significantly increased under fasted conditions only in males. Mature males consumed a higher amount of food but, as the OTA dose administered, it was proportional to body weight. The reason why the OTA bioavailability is more affected in presence of food only in males is unknown. Several possibilities, such as differences in gastric emptying, OTA-food interactions and the involvement of alpha-2u-globulin are discussed. 


\section{Introduction}

Ochratoxin A (OTA) is a mycotoxin produced by several species of Aspergillus and Penicillium which can contaminate a great variety of foodstuffs and to which humans are continuously exposed, although at very low doses. The main products contaminated with ochratoxin A are plant products such as cereals, coffee beans, beans, pulses and dried fruit (EFSA, 2006), but human exposure can also occur via the consumption of meat products from animals that have been fed with contaminated feed (NTP, 1989). The target organ of OTA is the kidney, inducing renal toxicity in all mammalian species tested and renal tumors in rodents. However, a wide range of other toxic effects, including hepatotoxicity (Bendele et al., 1985), immunotoxicity (Alvarez et al., 2004), teratogenicity (Pfohl-Leszkowicz and Manderville, 2007) and neurotoxicity (Belmadani et al., 1998; Sava et al., 2006), has also been described. In farm animals, it is considered to be a possible aetiological factor of some nephropathies in pigs (O'Brien and Dietrich, 2005) and in chickens (Stoev et al., 2002). In humans, it has been related with the Balkan Endemic Nephropathy (BEN) and with increases in the incidence of urinary tract tumors (Pfohl-Leszkowicz et al., 2002), but there is a lack of epidemiological evidence. Based on the aforementioned, the International Agency for Research on Cancer (IARC) classified OTA as a possible human carcinogen (group 2B), supported by sufficient evidence of carcinogenicity in animals studies and inadequate evidence in humans (IARC, 1993). A tolerable weekly intake (TWI) of $120 \mathrm{ng} / \mathrm{kg} \mathrm{b.w}$. has also been established by the European Food Safety Authority (EFSA, 2006).

However, although OTA has been thoroughly studied, there is still a concern regarding its potential genotoxicity and its mechanism of action as a carcinogen. A point that still remains unclear is the marked difference in sex specificity of OTA carcinogenicity in rodents (Bendele et al., 1985; NTP, 1989). The incidence of tumors in male rats is much higher than in females, but the mechanisms underlying this difference of sensitivity to OTA toxicity and the question of whether this is relevant or not for humans has still not been resolved. 
It is widely known that the main objective of toxicokinetic studies is to provide information on systemic exposure and its relationship to dose levels and toxicity. The inter-species differences in absorption, distribution, metabolism and excretion processes make it difficult to extrapolate animal data to predict human safety. With a better understanding of toxicokinetics and mechanisms of biological activity, it is possible to reduce the uncertainty regarding the extrapolation of animal toxicology data to predict human safety (Dixit et al., 2003). Unfortunately, in the main carcinogenesis studies performed to date with both sexes (NTP, 1989; Castegnaro et al., 1998; Son et al., 2003), OTA plasmatic concentration was not measured. Therefore, the impact of the toxicokinetics in OTA carcinogenesis was impossible to evaluate and to compare between the two genders. Recently, a kinetic study comparing young F344 rats (10 weeks old) and mature rats ( 15 weeks old) of both sexes (Vettorazzi et al., 2009) was carried out in order to evaluate the impact of sex and age on OTA toxicokinetics after a single oral dose. For the purpose of mimicking the conditions of longterm toxicological studies and natural exposure, the animals did not fast (fed conditions) before the single oral dose administered by gavage $\left(0.5 \mathrm{mg} / \mathrm{kg} \mathrm{b.w}\right.$. in $\mathrm{NaHCO}_{3} 0.1 \mathrm{M} \mathrm{pH}$ 7.4). This study showed a different kinetic profile in mature males, which presented a lower bioavailability and a higher apparent volume of distribution than in females and young rats. However, the apparent volume of distribution increased significantly with body weight. Therefore, the difference between genders obtained in this study could be related to malespecific mechanisms or to weight, because these two factors are not independent: for a same age, males always weigh more than females.

Even though administering OTA to animals under fed conditions was performed to approach long-term studies situation, it is known that food can alter bioavailability of substances by various means, including: delay gastric emptying, stimulate bile flow, change gastrointestinal $\mathrm{pH}$, increase splanchnic blood flow, change luminal metabolism of a substance, and physically or chemically interact with the substance (FDA, 2002). Numerous kinetic studies have been performed in order to evaluate food-effect in different substances, especially in 
pharmaceutical drugs. Some of the results obtained in these studies have been unpredictable, leading to a wide range of interactions that went from no effect to reduction, delay, increase or acceleration of the drug absorption (for a review see Welling, 1996). Even though the effect of standard laboratory diet in OTA absorption in rats has not been specifically studied, some studies have been performed in order to search for active adsorbents that would decrease OTA gastrointestinal absorption in animals (Huwig et al., 2001; Avantaggiato et al., 2007; Denli et al., 2008). Aoudia et al. (2008) described a protective effect against OTA absorption in rats when micronized wheat fibres (MWF) were added to feed contaminated with the mycotoxin. The authors attributed this effect to the fact that some of the plant cell wall components would trap the mycotoxin in their matrix, preventing toxin absorption from the gastrointestinal tract and favouring its elimination by faeces.

Another factor that may be involved in the kinetic differences between gender and age of rats is the presence of the male-rat-specific protein alpha-2u-globulin. The alpha-2u-globulin is a low molecular weight protein that is synthesized in the liver of males under androgenic control and reaches very high levels in plasma and urine of adult male rats. Many chemicals, such as d-limonene or 2,2,4-trimethylpentane, induce accumulation of the alpha-2u-globulin in the male kidney (for a review see Swenberg, 1993). This accumulation initiates a sequence of events that appears to lead to nephropathy and renal tumor formation (Rodgers and Baetcke, 1993). None of these chemicals have caused a similar nephropathy in female rats or in either sex of any other species (Swenberg, 1993). Even though it has been demonstrated that ochratoxin A kidney lesions are different from the alpha-2u-nephropathy in all characteristic points (Rasonyi et al., 1999) and that OTA also causes renal tumors in male mice (Bendele et al., 1985), a new concept involving this low protein in OTA toxicity has been published (Mantle and Nagy, 2008). These authors suggested that the alpha-2u-globulin may act as a specific OTA carrier that increases proximal tubule exposure to the mycotoxin in the male rat. 
For all the above mentioned reasons, the objective of this study was to evaluate the possible factors that may be in the origin of the different kinetic profile obtained in fed mature male rats described in (Vettorazzi et al., 2009). The first aspect that needed to be studied is the markedly lower OTA bioavailability obtained in mature males. For this purpose, and in order to determine if there was an interaction with food, a $24 \mathrm{~h}$ kinetic study in fasted conditions has been performed. Food ingestion in young and mature F344 rats of both sexes has also been controlled during two months. Finally, the presence of the male-specific protein alpha-2uglobulin in the urine of all of the different groups of animals has been studied.

\section{Materials and methods}

\subsection{Reagents}

OTA was purchased from Sigma (Steinheim, Germany). For the animal oral administration, OTA was dissolved in $0.1 \mathrm{M} \mathrm{NaHCO}_{3}$ (Sodium Hydrogen Carbonate powder. Riedel-deHaën, Seelze, Germany) and adjusted to $\mathrm{pH} 7.4$ with $\mathrm{HCl}$. OTA solutions were prepared in chemical safety cabinet and were always manipulated with double gloves and filtering masks FFP3. For the retro-orbital blood collection, animals were anesthetized with isoflurane (IsoFlo $^{\circledR}$, Veterinaria Esteve, Barcelona, Spain). All reagents used for the HPLC analysis were of pro-analysis grade. Sodium acetate and phosphoric acid were purchased from Panreac (Barcelona, Spain). Acetonitrile and methanol HPLC grade were obtained from Sigma Aldrich (Steinheim, Germany). The reagents used for OTA extraction and calibration standards were: absolute ethanol UV-IR-HPLC PAI and trichloroacetic acid $20 \% \mathrm{w} / \mathrm{v}$, both from Panreac (Barcelona, Spain) and normal saline solution 0.9\% from Braun (Barcelona, Spain). HPLC water from Millipore's Milli-Q System was used throughout the analysis.

The SDS-PAGE was performed on $10-15 \%$ gradient precast mini gels using SDS buffer strips (PhastGel ${ }^{\mathrm{TM}}$, GE Healthcare, Uppsala, Sweden). Loading buffer ( $\mathrm{pH}=8$ ) was $40 \mathrm{mM}$ Tris/HCl (Tris: PlusOne, Pharmacia Biotech, Buckinghamshire, UK), 4 mM EDTA (Sigma, St 
Louis, USA), 10\% SDS (PlusOne, Pharmacia Biotech, Uppsala, Sweden), 20\% $\beta$ mercaptoethanol (Sigma, St Louis, USA) and bromophenol blue sodium salt (Sigma, St Louis, USA). The coomassie stain used was Instant Blue ${ }^{\circledR}$ (Expedeon, Cambridge, UK). The positive control was male rat alpha-2u-globulin, same as used by Mantle and Nagy (2008). The molecular weight marker used was prepared from LMW-SDS marker kit (GE Healthcare, UK).

\subsection{Kinetic study}

\subsubsection{Animals}

All the animal studies performed were approved by the Ethics Committee on Animal Experimentation of the University of Navarra (Spain).

For the kinetic analysis, 15-week-old male $(n=18)$ and female $(n=18)$ Fisher $344(F 344)$ rats were used. All of the animals were purchased from Harlan (Horst, The Netherlands). On the day of arrival, the animals were weighed in order to assure that weight variation did not exceed $\pm 20 \%$ (OECD Guideline for testing of chemicals, Number 417 ). They were randomly housed in groups in polycarbonate cages with stainless steel covers so as to permit acclimatization to the environmental conditions (12h day/night cycle, temperature $22 \pm 2^{\circ} \mathrm{C}$, relative humidity $55 \pm$ 10\%, standard diet "2014 Teklad Global 14\% Protein Rodent Maintenance Diet" from Harlan Iberica Spain and water ad libitum) one week before commencing the study. On the day of administration, the animals were weighed (males: $259.6 \pm 7.9 \mathrm{~g}$ and females: $169.9 \pm 6.6 \mathrm{~g})$ in order to adjust the volume $(1 \mathrm{~mL} / 100 \mathrm{~g} \mathrm{b.w.})$ and the dose to be administered $\left(0.5 \mathrm{mg} / \mathrm{kg}\right.$ b.w. of OTA dissolved in $\left.\mathrm{NaHCO}_{3} 0.1 \mathrm{M} \mathrm{pH} 7.4\right)$ to the animal weight.

The experimental design was the same as in Vettorazzi et al. (2009) so as to allow comparison between the two studies. However, in this case, feed, but not water, was withdrawn $12 \mathrm{~h}$ before the administration. Animals were administered a single oral OTA dose 
(0.5 mg/kg b.w.) dissolved in $\mathrm{NaHCO}_{3}(0.1 \mathrm{M} \mathrm{pH} 7.4)$ by oral gavage and were denied feed until 8 hours after the administration. In order to determine OTA concentrations in plasma at $10 \mathrm{~min}, 30 \mathrm{~min}, 1 \mathrm{~h}, 2 \mathrm{~h}, 6 \mathrm{~h}$ and $8 \mathrm{~h}$, blood from 3 animals per timepoint was collected from the retro-orbital sinus under isoflurane anesthesia. Blood from each animal was extracted only once and the extraction volumes chosen were volumes that would not affect volaemia (Diehl et al., 2001). At $12 \mathrm{~h}$ and $24 \mathrm{~h}$ (in order to obtain kidneys and livers for future experiments), plasma was obtained by decapitation ( $\mathrm{n}=3$ per endpoint).

\subsubsection{Determination of OTA in plasma by HPLC-FLD}

OTA quantification was performed with a method specifically validated for OTA determination in plasma of rats (Vettorazzi et al., 2008). After $30 \mathrm{~min}$ at room temperature, $250 \mu \mathrm{L}$ of plasma were treated with $400 \mu \mathrm{L}$ of ice-cold absolute ethanol and $50 \mu \mathrm{L}$ of $20 \%$ trichloroacetic acid, vortexed for $1 \mathrm{~min}$, mixed in a rotating shaker for $15 \mathrm{~min}$ and centrifugated at $6200 \mathrm{~g}$ at $4{ }^{\circ} \mathrm{C}$ for $15 \mathrm{~min}$. Supernatants were injected into the HPLC after having been filtered with $0.45 \mu \mathrm{m} 13 \mathrm{~mm}$ Millex-HV syringe-driven polyvinylidene difluoride (PVDF) filter unit (Millipore, Bedford, USA). For blood samples with an expected high OTA concentration, supernatants were diluted $(1 / 20)$ in a solution $1: 5: 8(20 \%$ trichloroacetic acid: normal saline solution: absolute ethanol) before HPLC injection.

The analysis was performed with an Agilent Technologies 1100 liquid chromatographic system equipped with a fluorescence detector (model G1321A) controlled by Chemstation 3D software (Hewlett-Packard). OTA was analysed on a $5 \mu \mathrm{m}(25 \times 0.4 \mathrm{~cm})$ Tracer Extrasil ODS2 column and a Tracer Extrasil ODS-2 precolumn, both from Teknokroma (Barcelona, Spain). The mobile phase was 29:29:42 (v/v) methanol-acetonitrile-sodium acetate $(5 \mathrm{mM}$ acidified to $\mathrm{pH} 2.6$ with phosphoric acid). The aqueous phases were filtered through a 0.45 $\mu \mathrm{m}$ nylon membrane filter (Teknokroma, Barcelona, Spain). The injection volume was $100 \mu \mathrm{L}$ and the flow rate was $1.5 \mathrm{~mL} / \mathrm{min}$. Chromatography was performed at $40^{\circ} \mathrm{C}$ and monitored by 
fluorescence detection (excitation at $\lambda=225 \mathrm{~nm}$ and emission at $\lambda=461 \mathrm{~nm}$ ). The retention time for OTA in these conditions was $6.1 \mathrm{~min}$.

The LOD (limit of detection) and LOQ (limit of quantification) of this analytical method were 1 $\mu \mathrm{g} / \mathrm{L}$ and $8.4 \mu \mathrm{g} / \mathrm{L}$, respectively. Recovery percentage was very efficient $(96.0 \%)$ and the coefficient of variation (CV\%) obtained in within- and between-day experiments were below $10 \%$. All of the OTA concentrations obtained in samples have been corrected by the recovery value.

\subsubsection{Kinetic analysis}

The concentration versus time data obtained from all of the animals in both groups was analyzed simultaneously using the software NONMEM version VI (Beal et al., 1989-2006). The $24 \mathrm{~h}$ kinetic study in fasted conditions was performed in order to determine if the lower bioavailability obtained in fed mature males (Vettorazzi et al., 2009) was due to food interaction or to another cause (for example, a different first pass effect). Thus, in order to compare the results between the two studies, the extraction timepoints were exactly the same. This implies that in the current study, the majority of the animals contributed to the pharmacokinetic data with only one observation, preventing thereby the estimation of both inter-animal and residual variability. Consequently, the naïve pool approach (Garrido et al., 1999) was undertaken, in which all of the data is simultaneously analyzed, assuming it comes from a single animal. In this type of approach, only the parameters describing the mean pharmacokinetic profiles are estimated.

Based on these limitations in the data, it was decided that the typical population estimates of the apparent volumes of distribution of the central and peripheral compartments $(0.0276$ and $0.0158 \mathrm{~L}$, respectively), the apparent total and inter-compartmental clearances $\left(1.4 \times 10^{-4}\right.$ and $8.2 \times 10^{-4} \mathrm{~L} / \mathrm{h}$, respectively), the first order rate constant of absorption, $\mathrm{K}_{\mathrm{A}},\left(1.24 \mathrm{~h}^{-1}\right)$, and a relative bioavailability $(F)$ of 1 , all of which were obtained in the previous population 
pharmacokinetic analyses (Vettorazzi et al., 2009), would be used during the modelling process. We decided to focus only in differences between fed and fasted conditions in the parameters related to the absorption process, $\mathrm{K}_{\mathrm{A}}$, and $\mathrm{F}$.

The minimum value of the objective function provided by NONMEM, approximately equal to 2xlog (likelihood) [-2LL], was used to study the significance of food effects on $\mathrm{K}_{\mathrm{A}}$ and $\mathrm{F}$. For two nested models, a decrease in 3.84 , or 6.63 , points in $-2 \mathrm{LL}$ for an extra parameter was considered significant at the 5 or $1 \%$ levels, respectively.

\subsection{Food consumption analysis}

Food intake data from OTA treated (single oral administration, $0.5 \mathrm{mg} / \mathrm{kg}$ b.w. in $\mathrm{NaHCO}_{3}$ $0.1 \mathrm{M} \mathrm{pH} 7.4) \mathrm{F} 344$ rats were analyzed in order to study the differences between sexes and ages (10 weeks old and 15 weeks old).

According to the study design, animals $(n=32)$ were housed in cages in groups of 4 . Food intake of two cages of animals per experimental group (young males, young females, mature males and mature females) were controlled weekly for a period of 8 weeks ( $n=2$ cages $\times 8$ weeks $=16$ data per experimental group). The average intake of food per animal and day was then calculated for each cage.

The statistical analysis of food consumption data was performed with SPSS 15.0. The distribution of the data was checked for normality by the Shapiro-Wilk test and the homogeneity of the variance was verified by the Levene test. The results were analyzed by the two-way analysis of the variance (ANOVA), followed by Student's t-test. The alpha level was 0.05 . The between subject factors were: age (10 weeks old and 15 weeks old) and sex. However, it must be taken into account that the two groups of ages compared statistically correspond to two intervals of ages: from 10 weeks old to 18 weeks old for the rats that were 10 weeks old at the beginning of the study, and the interval from 15 weeks old to 23 weeks old for the rats that were 15 weeks old at the beginning of the study. 
Finally, in order to avoid the influence of the animal body weight in food consumption, the intake of food was normalized by $100 \mathrm{~g}$ of body weight, and another two-way ANOVA was conducted.

\subsection{Alpha-2u-globulin study}

In order to compare the presence of alpha-2u-globulin, urines of young male, young female, mature male and mature female F344 rats were analyzed.

For the comparison between males and females at different ages (10 weeks old and 15 weeks old), $24 \mathrm{~h}$ collection of urine of non-treated rats were studied ( $\mathrm{n}=2$ per group) (gel 1). In order to compare the 10-week-old and 15-week-old OTA treated males (gel 2), urine collected during the first $24 \mathrm{~h}$ after a single oral dose of OTA $\left(0.5 \mathrm{mg} / \mathrm{kg}\right.$ b.w. in $\mathrm{NaHCO}_{3} 0.1$ $\mathrm{M} \mathrm{pH}, 7.4$ ) was analysed ( $\mathrm{n}=3$ per group).

The alpha-2u-globulin analysis was performed using PhastSystem ${ }^{\mathrm{TM}}$ (Pharmacia). The urine samples were centrifugated for $5 \mathrm{~min}$ at $14000 \mathrm{~g}$ in order to remove impurities. Then, $5 \mu \mathrm{L}$ of urine was diluted $1 / 2$ with loading buffer and heated in thermoblock at $88^{\circ} \mathrm{C}$ for $5 \mathrm{~min}$. The alpha-2u-globulin control was diluted $1 / 10$ with loading buffer and also heated at $88^{\circ} \mathrm{C}$ for 5 min. Then according to manufacturer's protocol, $1 \mu \mathrm{L}$ of each sample was loaded on the precast gradient $10-15 \% \mathrm{gel}$ and run for $20 \mathrm{~min}$. The gels were stained with Instant Blue $囚$ and the image was captured by the Syngene (Cambridge, UK) Diversity imaging system.

\section{Results}

After the single oral administration, OTA plasmatic levels were measurable in both sexes at all timepoints. Mature males presented a maximum observed OTA concentration $\left(\mathrm{CMAX}_{\mathrm{obs}}\right)$ of $4148.9 \mu \mathrm{g} / \mathrm{L}$ at $2 \mathrm{~h}$, while females reached a CMAX obs $3674.4 \mu \mathrm{g} / \mathrm{L} 6$ hours after the administration. Figure 1 shows the plasma concentration expressed as mean values of three observations per timepoint together with the corresponding standard deviation, in mature 
males and females rats after a single oral administration of ochratoxin $A$ in fasted conditions. Figure 1 also shows the predicted profiles corresponding to the model selected and parameters estimated from data obtained in young and mature rats after a single oral administration of ochratoxin $A$ in fed conditions, where it was found that male mature rats showed a $34 \%$ decrease in relative bioavailability with respect to young males and females (young and mature) (Vettorazzi et al., 2009). Comparing the observed data under fasted conditions with the model predicted kinetic profile under fed conditions, it was observed that relative bioavailability in females is equivalent under both conditions. However, in the case of the males, F in fasted conditions is clearly increased with respect to the presence of food. With respect to $F$, fasted mature male and fasted mature female rats did not differ between themselves nor did they differ from young males and females (young and mature) in fed conditions $(p>0.05)$. Therefore, the relative bioavailability of fasted mature males increased $34 \%$ with respect to fed mature males. In addition, it appears that the rate of absorption is higher for both males and females in fasted conditions, since at early times after administration, observations appeared under-predicted by the model developed based on animal fed data. The value of $\mathrm{K}_{\mathrm{A}}$ resulted significantly higher $(p<0.01)$ in fasted conditions $\left(5.24(15.3 \%) h^{-1}\right)$ compared with the animals with free access to food $\left(1.24(14 \%) h^{-1}\right)$ (Vettorazzi et al., 2009). This analysis could also identify a small lag time in the absorption process of $8.5(4.1) \mathrm{min}$.

The kinetic model selected for this study (solid lines in figure 1) has been obtained by updating the model previously selected in fed animals with the new estimates of $\mathrm{K}_{\mathrm{A}}$, lag time, and considering $\mathrm{F}$ to be equal to one in both males and females. For comparison, the profiles obtained only with fed animals are superimposed as dashed lines in figure 1.

Food ingestion data of rats of both sexes at different ages is presented in figure $2 \mathrm{~A}$. In order to evaluate the effect of age and sex on food ingestion, a two-way ANOVA was conducted. The two factors studied were age (10 weeks old and 15 weeks old) and sex. There was an 
interaction between the two factors $\left[F_{1}=38.840, p<0.001\right]$. Further analysis showed that young animals ingested less food than mature animals in both sexes (Student's t-test, $p$ $<0.001$ ) and that for the same age, males consumed more food than females (Student's ttest, $p<0.001)$.

However, when food ingestion was normalized by $100 \mathrm{~g}$ of body weight (figure 2B) the differences between groups disappeared. After performing the two-way ANOVA, no interaction was found between the two groups $\left[F_{1}=0.808, p>0.05\right]$ and no significant main effect was found for sex $\left[F_{1}=0.808, p>0.05\right]$ or age $\left[F_{1}=0.600, p>0.05\right]$.

With regard to the alpha-2u-globulin study, females of all ages did not eliminate alpha-2uglobulin in urine, whereas alpha-2u-globulin it was present in the urine of males of all ages (figure 3: gel 1). In gel 2 (figure 3), urine from OTA-treated males of both ages were compared. No apparent differences between 10-week-old and 15-week-old males were observed. The differences of intensity of the bands were inversely correlated with the volume urinated (table of figure 3).

\section{Discussion}

The results obtained in the present study indicate that OTA relative bioavailability in mature males is much more influenced by food that in the case of females. The administration of OTA by oral gavage in fasted conditions, when comparing to fed conditions (Vettorazzi et al., 2009), tend to increase the $\mathrm{CMAX}_{\mathrm{obs}}$ and the rate of absorption in all of the groups while not changing time at maximum observed concentration $\left(\operatorname{Tmax}_{\mathrm{obs}}\right)$. These results indicate that food does not appear to delay the absorption window but seems to affect the extent of OTA absorption. However, only mature males in fed conditions showed a statistically significant difference (34\% less relative bioavailability than the rest of the groups).

After monitoring the food intake for 2 months in all of the groups, a statistical difference was detected among all groups, being the consumption in mature males $>$ young males $>$ mature 
females $>$ young females. This pattern of food consumption is inversely related with the OTA plasma levels describing the kinetic curves of each group in fed conditions: mature males < young males < mature females < young females (Vettorazzi et al., 2009). However, only the kinetic curve of mature males was significantly different from the rest of the groups. When food consumption was normalized by $100 \mathrm{~g}$ of body weight, differences between the different groups disappeared. Taking into account that OTA has been administered in each case, adjusting the dose to body weight $(0.5 \mathrm{mg} / \mathrm{kg}$. b.w), the amount of OTA by grams of food in the GI tract of each animal should be the equivalent, and therefore the trapping effect that food may exert over the mycotoxin should be the same in both genders at the different ages. Therefore, the major difference regarding food consumption may lie in the higher absolute quantity of food ingested by mature males and/or in the anatomo-physiological differences in the GI tract between genders in mature animals.

In general, a high volume of food normally delays gastric emptying (Shargel et al., 2005). It is also known that OTA is mainly absorbed in the upper part of the GI tract between stomach and proximal jejunum (Kumagai and Aibara, 1982; Pfohl-Leszkowicz and Manderville, 2007). If an anatomic absorption window exists within the GI tract in which OTA is efficiently absorbed (Shargel et al., 2005), it could be hypothesized that the differences in gastric emptying may account for the less efficient absorption profile observed in mature males in fed conditions. Normally, a delay in the gastric emptying slows the rate and the extent of the absorption because it also delays the time for a substance to reach the small intestine (Shargel et al., 2005). The results of this experiment indicate that this could also be happening with OTA.

Moreover, a higher amount of food and a delay in the gastric emptying could also allow a larger contact in the stomach of OTA with the components of the food that may have a trapping effect towards the mycotoxin. One of the main ingredients of the pellet feed given to the animals is wheat and the total content of fibre in the pellets is 4.50\% (2014 Teklad Global 
14\% Protein Rodent Maintenance Diet from Harlan). Aoudia et al. (2008) clearly demonstrated that micronized wheat fibers mixed in the animal diet act as active adsorbents. They demonstrated the protective effect of wheat fibers in terms of OTA concentration in plasma, kidney and liver. Thus, it seems plausible that a higher amount of food and/or a higher time in contact with food in the stomach, may lead to less OTA absorption.

This coincides with the study performed by Mantle et al. (2005), where they observed that OTA had significantly less potency as a renal carcinogen in male rat when acquired as a dietary component than when given once daily by oral gavage (NTP, 1989). Furthermore, Miljkovic et al. (2003) also pointed out that the route of administration is a critical factor. These authors found that OTA plasma levels were lower when administered via diet than when administering the same doses ( $0.2 \mathrm{mg}$ and $1 \mathrm{mg}$ OTA) by gavage, with the OTA plasma concentration being much lower in the animals receiving the higher dose by diet. Moreover, for the same dose of OTA, lesions were observed in the gavage-administered animals while there was an absence of pathology in the animals that were administered the equivalent dietary dose.

The possible effect of the volume of food in the stomach coincides with the hypothesis that OTA is mainly passively absorbed from the GI (reviews Ringot et al. 2006; Pfohl-Leszkowicz and Manderville, 2007). However, the possibility of a carrier-mediated transport must not be discarded. Dietrich et al. (2005) suggested the presence of organic anion transporters with the capacity to transport OTA in the jejunum, but Berger et al. (2003) showed that these transporters were not implicated in OTA transport across Caco-2-cells. Another factor that may influence the OTA bioavailability is the finding that OTA is a substrate for the ATPBinding Cassette efflux-proteins MRP2 and BCRP in Caco-2-cells (Schrickx et al., 2006). These ATP-dependent membrane transporters facilitate secretion of xenobiotics outside the cell, thus, counteracting the absorption of toxic compounds (Schrickx et al., 2006). These authors also suggested that OTA bioavailability may also vary in the presence of food 
because many food components are BCRP substrates. Moreover, Tanaka et al. (2005) found differences between genders in BCRP expression in different tissues of Sprague-Dawley rats. BCRP mRNA levels were high in male kidney, small intestine and large intestine, but were only statistically different from females in kidney. Even if these experiments were performed in another rat strain and the animals were much younger than the one used in our study, the influence of sex hormones in the expression of these receptors has been demonstrated.

Other factors, such as changes in $\mathrm{pH}$ of the $\mathrm{Gl}$ tract, stimulation of bile flow, increase in splanchnic blood flow or changes in the luminal metabolism that may be affected by the presence of food in the stomach must be also taken into account (FDA, 2002). However, even if the highest amount of food could be a cause, the exact reason of why food intake affects mature males much more than the rest of the groups needs to be obtained with mechanistic studies due to the lack of published data regarding anatomical and physiological differences between genders in rats.

The experimental design in this study was performed in order to make it comparable with the study performed by Vettorazzi et al. (2009). For this reason, each animal only contributed with one datapoint, preventing the performance of a population approach modelling. Therefore, the kinetic analysis was focused only on differences between fed and fasted conditions for the parameters related to the absorption process $\left(K_{A}\right.$ and $\left.F\right)$. We doublechecked the results obtained in this study by simultaneously analyzing them with the data obtained in Vettorazzi et al. (2009) using the same population modeling approach strategy as in the previous study. The results from this re-analysis are shown in figure 4 and the new parameter estimates are presented in table 1, together with the parameters obtained in the study previously performed with fed animals only (Vettorazzi et al., 2009). This re-analysis confirmed the lower bioavailability of mature males in fed conditions that could be attributable to a lower OTA absorption in the presence of food. The relationship between V/F and weight 
was not significantly different from the one previously reported in fed animals (Vettorazzi et al., 2009).

The final experiment performed in this study was to determine a possible influence of the specific male rat protein alpha-2u-globulin in the kinetics of OTA. As expected, the protein was not present in urine of females at any of the two ages (Swenberg, 1993). The protein was present in males of the two ages studied, showing that in this case, the presence of alpha-2u-globulin might not be the cause of the different kinetic profile obtained in mature males in (Vettorazzi et al., 2009). However, our combined pharmacokinetic findings need not conflict with a concept of augmented uptake of OTA into nephron epithelia by binding to an androgen-dependent $\alpha_{2 u}$-globulin (Mantle and Nagy, 2008). The fact that fed mature males alone deviated from the general pattern of OTA pharmacokinetics could be consistent with glomerular filtration escape of some of the early-absorbed OTA carried on $\alpha_{2 u}$-globulin during the first few hours after gavage dosing. In any case, our electrophoretic demonstration of marked gender difference in incidence of $\alpha_{2 u}$-globulins in urine is only a distant qualitative reflection of the cryptic cycle of hepatic synthesis and fairly efficient renal salvage. The complexity of the $<20 \mathrm{kDa}$ urinary proteins of adult male rats has been revealed by twodirectional electrophoresis (Wait et al 2001) but little is known of their dynamics as male rats become adult. Further studies, such as proteomics and kinetic studies in the kidney would be needed to consolidate the concept of alpha-2u-globulin involvement in OTA toxicity.

In conclusion, the results obtained in this study clearly show that, when administered with food in the stomach, ochratoxin A bioavailability is lower, with the mature males being much more affected than females and young animals. This result highlights the need of taking into account the route of administration (gavage or dietary), the fast/fed conditions, the age and the gender of the animals used when interpreting the in vivo toxicological data generated to date. Furthermore, this finding might not only be important when studying OTA toxic effects in laboratory animals, but it could also be also relevant when estimating the OTA human 
exposure due to the wide variety of foodstuff susceptible of being contaminated with OTA. The physiological changes of the $\mathrm{Gl}$ tract and the trapping effect of the lumen content may vary depending on the food matrix in which the mycotoxin is present, and thereby affecting the mycotoxin bioavailability in different ways. Finally, it is also important to point out that the inter-gender differences and the influence of food observed in this study are not only relevant issues in ochratoxin A toxicity, but are also very interesting factors to be considered in any other chemical risk assessment.

\section{Appendix}

Supplementary data associated with this article, such as plasma concentrations raw data and kinetics parameters of each animal group, can be found in the online version.

\section{Funding}

This work was supported by the "Ministerio de Educación y Ciencia" of Spain [Ref. AGL200402942] and by the "Ministerio de Ciencia e Innovación" [Ref. AGL2008-01437]. Ariane Vettorazzi, Leire Arbillaga and Laura-Ana Corcuera thank the "Departamento de Educación, Universidades e Investigación del Gobierno Vasco" [Ref. BFI06.107 to A.V.], the "Ministerio de Educación y Ciencia" of Spain [Ref.BES-2005-9939 to L.A.] and the University of Navarra-Fundación Caja Navarra [LA.C.] for the grants received.

\section{Conflict of interest statement}

There is no conflict of interest.

\section{Acknowledgments}

The authors wish to express their gratitude to Ms. Laura Stokes for reviewing the English version of this manuscript. 


\section{References}

Alvarez, L., Gil, A.G., Ezpeleta, O., Garcia-Jalon, J.A. and Lopez de Cerain, A., 2004. Immunotoxic effects of Ochratoxin A in Wistar rats after oral administration. Food Chem. Toxicol. 42, 825-834.

Aoudia, N., Tangni, E.K. and Larondelle, Y., 2008. Distribution of ochratoxin A in plasma and tissues of rats fed a naturally contaminated diet amended with micronized wheat fibres: Effectiveness of mycotoxin sequestering activity. Food Chem. Toxicol. 46, 871-878.

Avantaggiato, G., Havenaar, R. and Visconti, A., 2007. Assessment of the multi-mycotoxinbinding efficacy of a carbon/aluminosilicate-based product in an in vitro gastrointestinal model. J. Agric. Food Chem. 55, 4810-4819.

Beal, S.L., Sheiner, L.B. in NONMEM User's guide (Icon Development Solutions, Ellicot City, Maryland, USA, 1989-2006).

Belmadani, A., Tramu, G., Betbeder, A.M., Steyn, P.S. and Creppy, E.E., 1998. Regional selectivity to ochratoxin A, distribution and cytotoxicity in rat brain. Arch. Toxicol. 72, 656662.

Bendele, A.M., Carlton, W.W., Krogh, P. and Lillehoj, E.B., 1985. Ochratoxin A carcinogenesis in the (C57BL/6J X C3H)F1 mouse. J. Natl. Cancer Inst. 75, 733-742.

Berger, V., Gabriel, A.F., Sergent, T., Trouet, A., Larondelle, Y. and Schneider, Y.J., 2003. Interaction of ochratoxin A with human intestinal Caco-2 cells: possible implication of a multidrug resistance-associated protein (MRP2). Toxicol. Lett. 140-141, 465-476.

Castegnaro, M., Mohr, U., Pfohl-Leszkowicz, A., Esteve, J., Steinmann, J., Tillmann, T., Michelon, J. and Bartsch, H., 1998. Sex- and strain-specific induction of renal tumors by ochratoxin A in rats correlates with DNA adduction. Int. J. Cancer 77, 70-75.

Denli, M., Blandon, J.C., Guynot, M.E., Salado, S. and Perez, J.F., 2008. Efficacy of a new ochratoxin-binding agent (OcraTox) to counteract the deleterious effects of ochratoxin $A$ in laying hens. Poult. Sci. 87, 2266-2272.

Diehl, K.H., Hull, R., Morton, D., Pfister, R., Rabemampianina, Y., Smith, D., Vidal, J.M., van de Vorstenbosch, C. and European Federation of Pharmaceutical Industries Association and European Centre for the Validation of Alternative Methods, 2001. A good practice guide to the administration of substances and removal of blood, including routes and volumes. J. Appl. Toxicol. 21, 15-23.

Dietrich, D.R., Heussner, A.H. and O'Brien, E., 2005. Ochratoxin A: comparative pharmacokinetics and toxicological implications (experimental and domestic animals and humans). Food Addit. Contam. 22 Suppl 1, 45-52.

Dixit, R., Riviere, J., Krishnan, K. and Andersen, M.E., 2003. Toxicokinetics and physiologically based toxicokinetics in toxicology and risk assessment. J. Toxicol. Environ. Health B Crit. Rev. 6, 1-40. 
EFSA (European Food Safety Authority), 2006. Opinion of the Scientific Panel on contaminants in the food chain on a request from the Commission related to ochratoxin $A$ in food. Adopted on 4 April 2006. The EFSA Journal 365, 1-56.

Food and Drug Administration. U.S Department of Health and Human Services. Center for Drug evaluation and research (CDER), 2002. Guidance for industry. Food-effect bioavailability and fed bioequivalence studies.

Garrido, M.J., Valle, M., Calvo, R. and Troconiz, I.F., 1999. Altered plasma and brain disposition and pharmacodynamics of methadone in abstinent rats. J. Pharmacol. Exp. Ther. 288, 179-187.

Huwig, A., Freimund, S., Kappeli, O. and Dutler, H., 2001. Mycotoxin detoxication of animal feed by different adsorbents. Toxicol. Lett. 122, 179-188.

IARC, 1993. Ochratoxin A. Some naturally occurring substances: food items and constituents, heterocyclic aromatic amines and mycotoxins. IARC Monographs on the evaluation of carcinogenic risks to humans $56,489-521$.

Kumagai, S. and Aibara, K., 1982. Intestinal absorption and secretion of ochratoxin A in the rat. Toxicol. Appl. Pharmacol. 64, 94-102.

Mantle, P. and Nagy, J., 2008. Binding of a Ochratoxin A to a Urinary Globulin: A New Concept to Account for Gender Difference in Rat Nephrocarcinogenic Responses. Int. J. Mol. Sci. 9, 719-735.

Mantle, P., Kulinskaya, E. and Nestler, S., 2005. Renal tumourigenesis in male rats in response to chronic dietary ochratoxin A. Food Addit. Contam. 22 Suppl 1, 58-64.

Miljkovic, A., Pfohl-Leszkowicz, A., Dobrota, M. and Mantle, P.G., 2003. Comparative responses to mode of oral administration and dose of ochratoxin A or nephrotoxic extract of Penicillium polonicum in rats. Exp. Toxicol. Pathol. 54, 305-312.

National Toxicology Program (NTP), 1989. Toxicology and carcinogenesis studies of ochratoxin A (CAS No 303-47-9) in F344/N rats (gavage studies). Nat. I Toxicol. Program Tech. Rep. Ser. 358, 1-142.

O'Brien, E. and Dietrich, D.R., 2005. Ochratoxin A: the continuing enigma. Crit. Rev. Toxicol. 35, 33-60.

OECD Guideline for testing of chemicals, Number 417. Adoped 4 April 1984., Toxicokinetics.

Pfohl-Leszkowicz, A. and Manderville, R.A., 2007. Ochratoxin A: An overview on toxicity and carcinogenicity in animals and humans. Mol. Nutr. Food Res. 51, 61-99.

Pfohl-Leszkowicz, A., Petkova-Bocharova, T., Chernozemsky, I.N. and Castegnaro, M., 2002. Balkan endemic nephropathy and associated urinary tract tumours: a review on aetiological causes and the potential role of mycotoxins. Food Addit. Contam. 19, 282-302.

Rasonyi, T., Schlatter, J. and Dietrich, D.R., 1999. The role of alpha2u-globulin in ochratoxin A induced renal toxicity and tumors in F344 rats. Toxicol. Lett. 104, 83-92. 
Ringot, D., Chango, A., Schneider, Y.J. and Larondelle, Y., 2006. Toxicokinetics and toxicodynamics of ochratoxin A, an update. Chem. Biol. Interact. 159, 18-46.

Rodgers, I.S. and Baetcke, K.P., 1993. Interpretation of male rat renal tubule tumors. Environ. Health Perspect. 101 Suppl 6, 45-52.

Sava, V., Reunova, O., Velasquez, A., Harbison, R. and Sanchez-Ramos, J., 2006. Acute neurotoxic effects of the fungal metabolite ochratoxin-A. Neurotoxicology 27, 82-92.

Schrickx, J., Lektarau, Y. and Fink-Gremmels, J., 2006. Ochratoxin A secretion by ATPdependent membrane transporters in Caco-2 cells. Arch. Toxicol. 80, 243-249.

Shargel, L., Wu-Pong, S. and Yu, A., 2005. Applied Biopharmaceutics and Pharmacokinetics, 5th Edition ed. McGraw-Hill Companies Inc.

Son, W.C., Kamino, K., Lee, Y.S. and Kang, K.S., 2003. Strain-specific mammary proliferative lesion development following lifetime oral administration of ochratoxin A in DA and Lewis rats. Int. J. Cancer 105, 305-311.

Stoev, S.D., Daskalov, H., Radic, B., Domijan, A.M. and Peraica, M., 2002. Spontaneous mycotoxic nephropathy in Bulgarian chickens with unclarified mycotoxin aetiology. Vet. Res. 33, 83-93.

Swenberg, J.A., 1993. Alpha 2u-globulin nephropathy: review of the cellular and molecular mechanisms involved and their implications for human risk assessment. Environ. Health Perspect. 101 Suppl 6, 39-44.

Tanaka, Y., Slitt, A.L., Leazer, T.M., Maher, J.M. and Klaassen, C.D., 2005. Tissue distribution and hormonal regulation of the breast cancer resistance protein (Bcrp/Abcg2) in rats and mice. Biochem. Biophys. Res. Commun. 326, 181-187.

Vettorazzi, A., Gonzalez-Peñas, E., Troconiz, I.F., Arbillaga, L., Corcuera, L.A., Gil, A.G. and de Cerain, A.L., 2009. A different kinetic profile of ochratoxin A in mature male rats. Food Chem. Toxicol. 47, 1921-1927.

Vettorazzi, A., Gonzalez-Peñas, E., Arbillaga, L., Corcuera, L.A. and Lopez de Cerain, A., 2008. Simple high-performance liquid chromatography-fluorescence detection method for plasma, kidney and liver of rat as a tool for toxicology studies. J. Chromatogr. A 1215, 100106.

Wait, R., Gianazza, E., Eberini, I. Sironi, L., Dunn, M.J., Gemeiner, M., Miller, I., 2001. Proteins of rat serum, urine, and cerebrospinal fluid: VI. Further protein identifications and interstrain comparison. Electrophoresis 22, 3043-3052.

Welling, P.G., 1996. Effects of food on drug absorption. Annu. Rev. Nutr. 16, 383-415. 
Figure 1. Plasma OTA concentrations [expressed as mean values (solid circles) together with the corresponding standard deviation (vertical lines)] in mature male and female rats after a single oral administration of ochratoxin $\mathrm{A}\left(0.5 \mathrm{mg} / \mathrm{kg}\right.$ b.w dissolved in $\mathrm{NaHCO}_{3} 0.1 \mathrm{M}$ $\mathrm{pH}$ 7.4) in fasted conditions. Solid lines show model predictions based on optimized model parameters obtained in the current study. Dashed lines correspond to the predicted profiles of a model previously obtained in young and mature rats after a single oral administration of ochratoxin A in fed conditions (Vettorazzi et al., 2009).

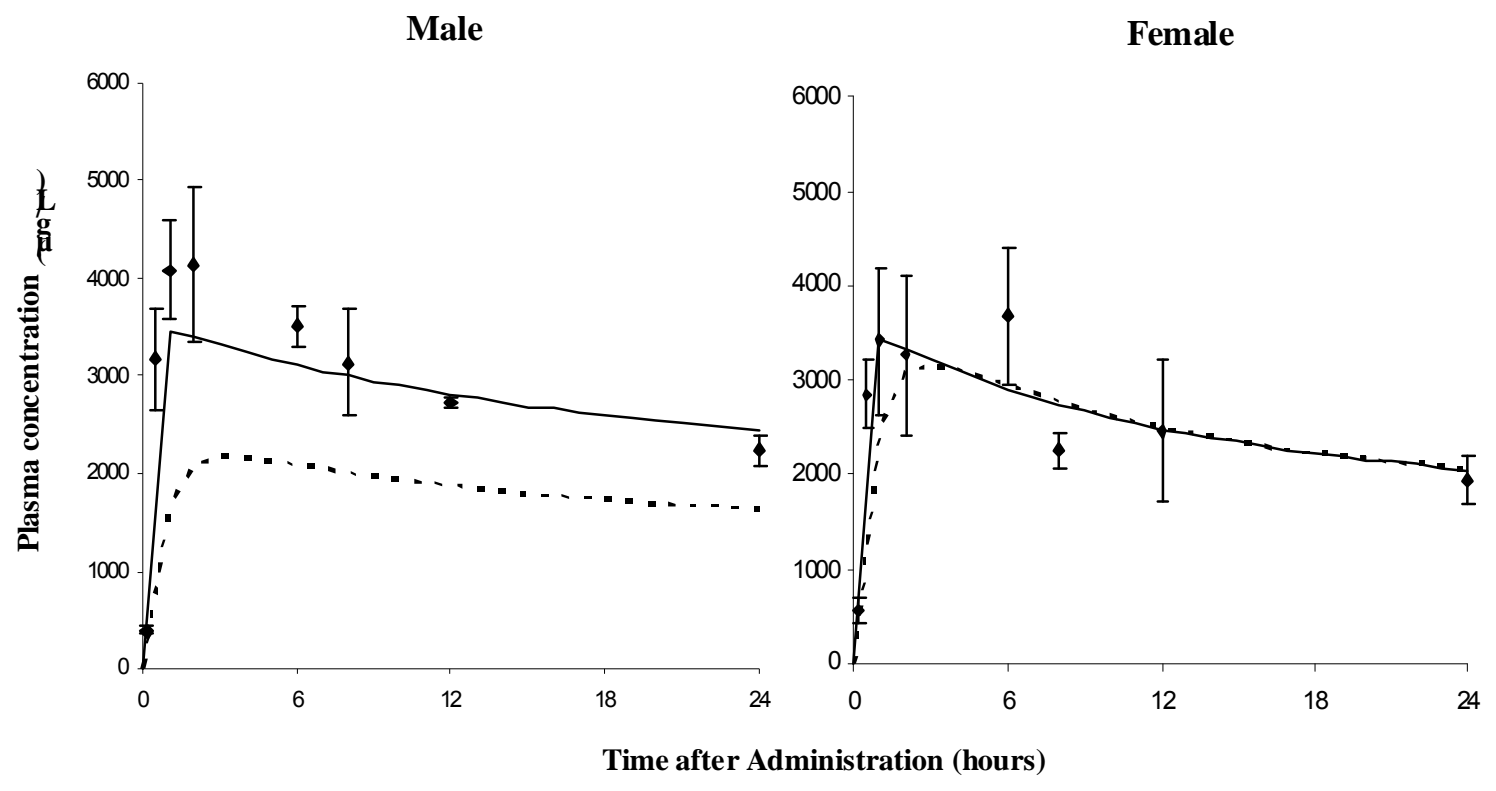


Figure 2. Means and standard deviations of total food consumption (2A) and food consumption per $100 \mathrm{~g}$ of b.w.of animal (2B).

2A)

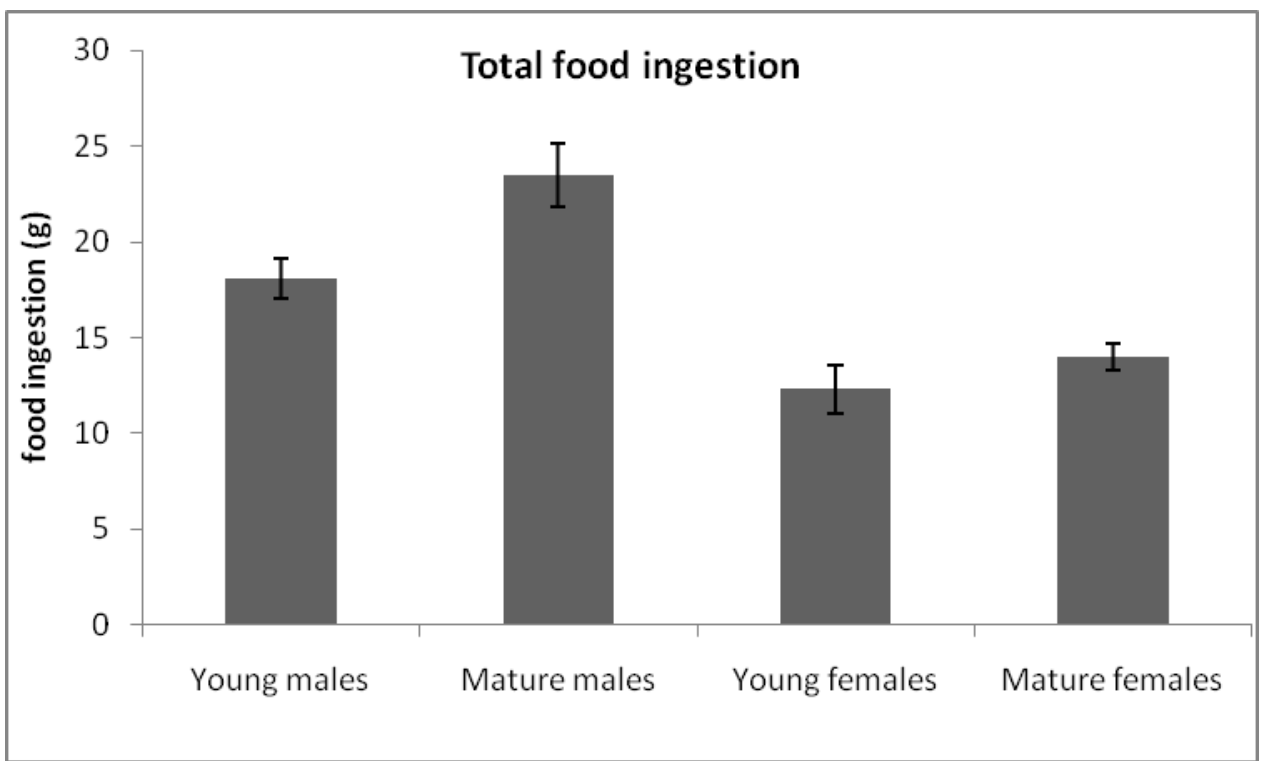

2B)

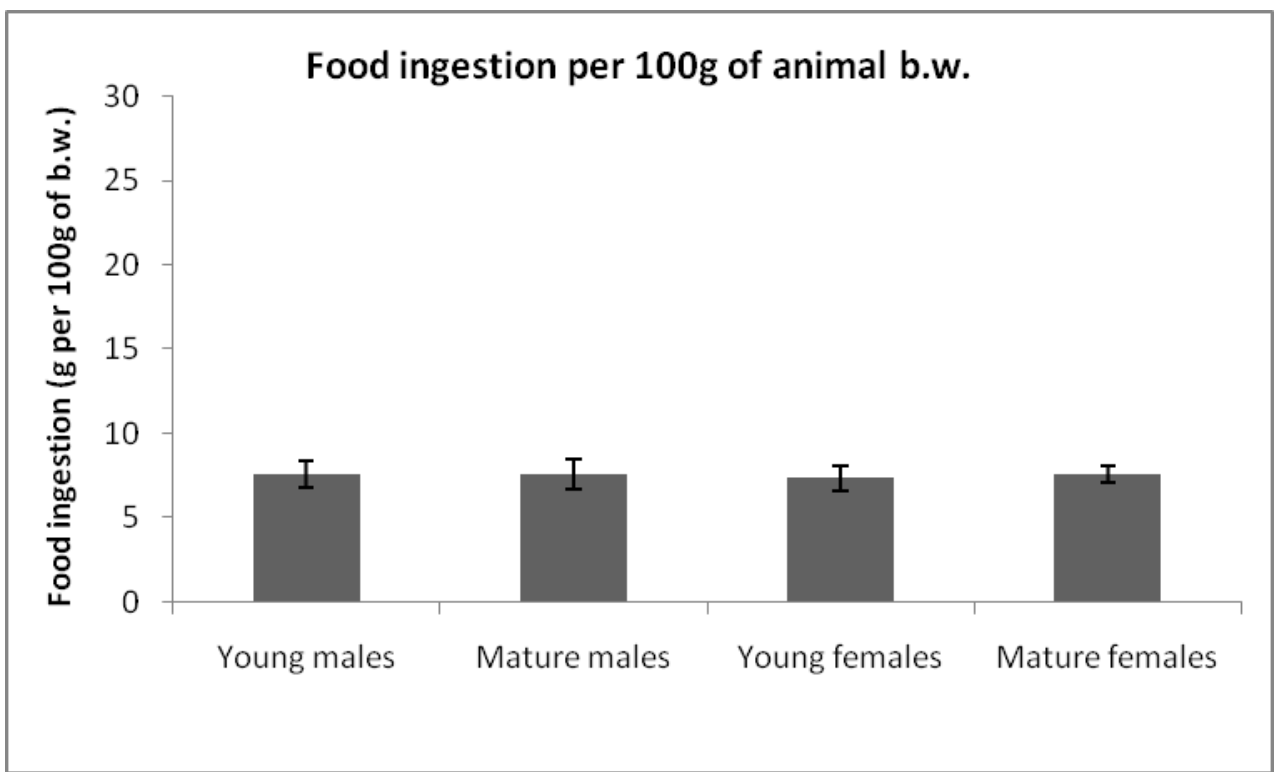


Figure 3. Coomassie-stained SDS-PAGE of urinary proteins of young and mature F 344 rats of both sexes performed in PhastGel gradient 10-15®. a2u: alpha-2u-globulin standard. MW: molecular weight marker. The table below shows the volumes of the $24 \mathrm{~h}$ collection of urine of young and mature F344 rats of both sexes used for the alpha-2u-globulin analysis.

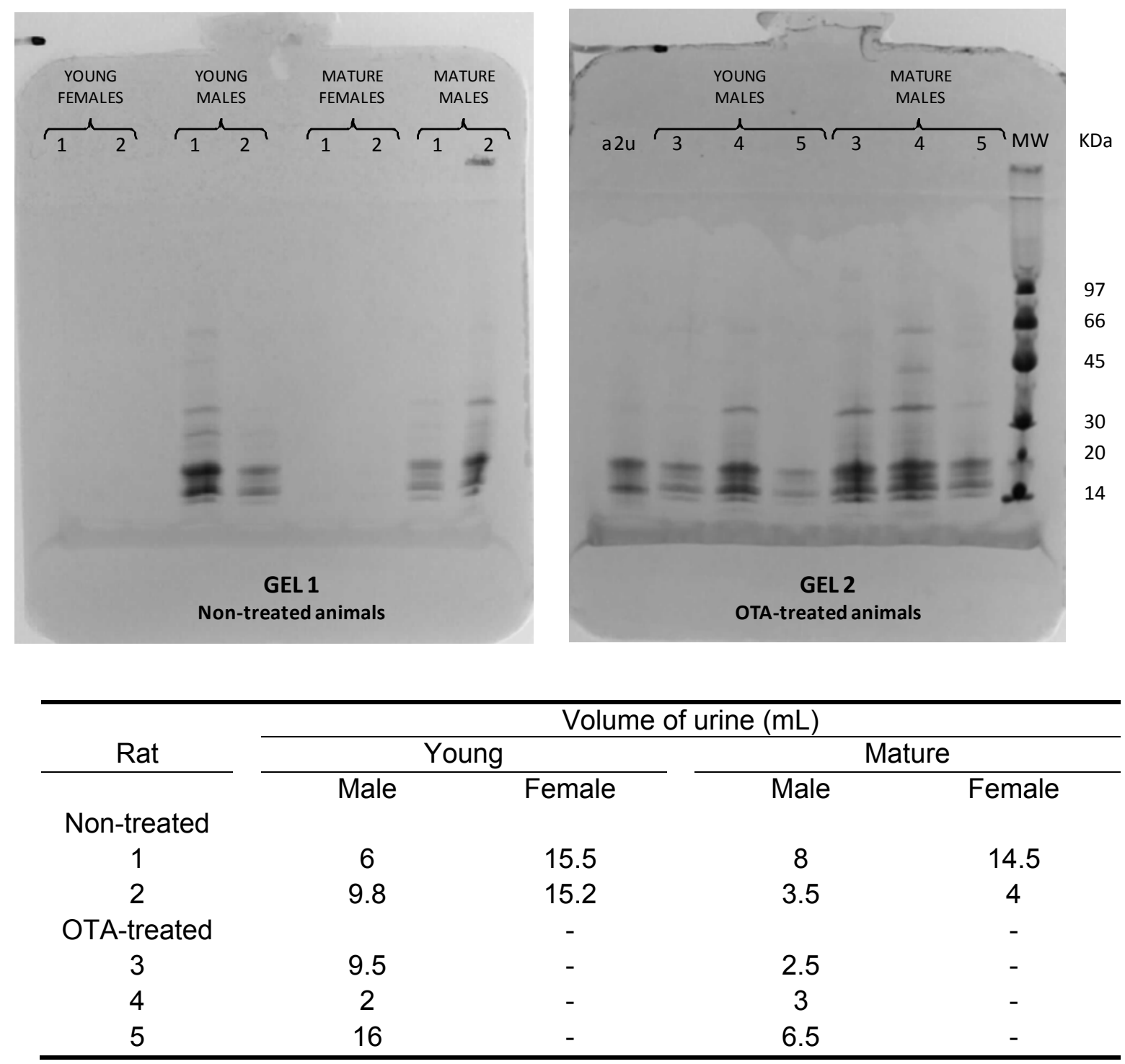


Figure 4. Evaluation of model performance of the selected population model. For each panel, open circles represent observations and lines show results from 1000 simulated profiles: solid $50^{\text {th }}$ percentile, dashed, 5 and $95^{\text {th }}$ percentiles.

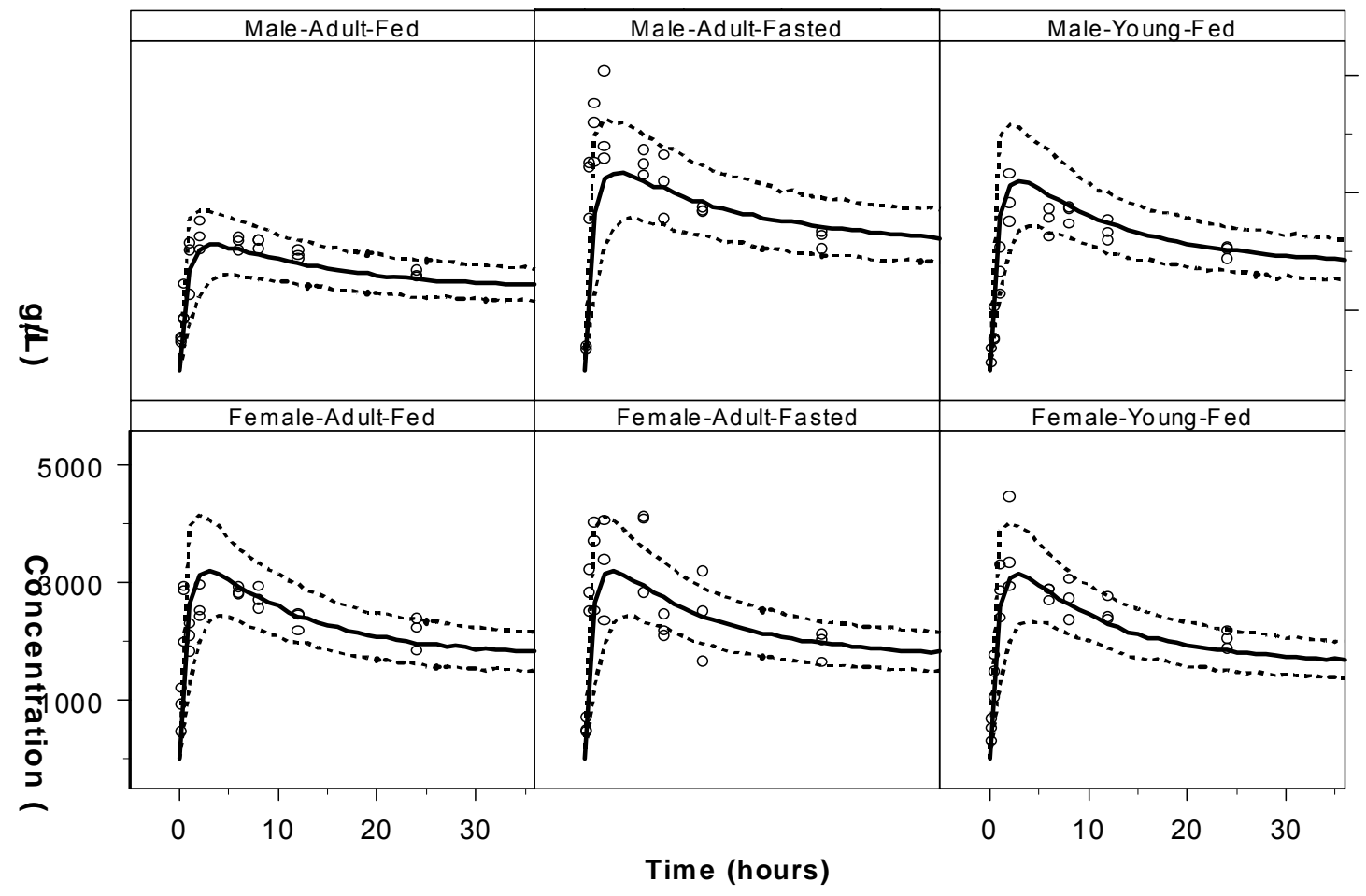


Table 1. Population pharmacokinetic model parameters estimates of the selected model of fed animals (young and mature animals of both sexes) (left column) and of the re-analysis that included fasted animals (right columns).

\begin{tabular}{ccc}
\hline Parameter & Fed animals & $\begin{array}{c}\text { Fed and Fasted } \\
\text { animals }\end{array}$ \\
\hline $\mathrm{K}_{\mathrm{A}}\left(\mathrm{h}^{-1}\right)$ & $1.24(14)$ & $1.26(12)$ \\
$\mathrm{V} / \mathrm{F}(\mathrm{L})$ & $0.0276(7)$ & $0.0255(5)$ \\
$\mathrm{CL} / \mathrm{F}\left(\mathrm{L} \times \mathrm{h}^{-1}\right)$ & $1.41 \times 10^{-4}(2)$ & $1.4 \times 10^{-4}(2)$ \\
$\mathrm{Q} / \mathrm{F}\left(\mathrm{L} \times \mathrm{h}^{-1}\right)$ & $8.2 \times 10^{-4}(28)$ & $1.15 \times 10^{-4}(15)$ \\
$\mathrm{V}_{\mathrm{T}} / \mathrm{F}(\mathrm{L})$ & $0.0158(10)$ & $0.0181(7)^{\mathrm{a}}$ \\
$\mathrm{F}$ & $1 *$ & $1 *$ \\
$\mathrm{~F}_{\text {Male\&Maturet\&Fed }}$ & $\mathbf{0 . 6 6 ( 2 . 4 )}$ & $\mathbf{0 . 6 4 ( 2 . 3 )}$ \\
IAV_k $\mathrm{k}_{\mathrm{A}}(\%)$ & $57(24)$ & $66(24)$ \\
IAV_V (\%) & $10(36)$ & $13(36)$ \\
IAV_F (\%) & - & - \\
Corr(h, $\left.\mathrm{h}_{\mathrm{F}}\right)$ & - & $10.5(16)$ \\
Residual $(\%)$ & $10(16)$ & -
\end{tabular}

Estimates are listed together with the corresponding coefficient of variation $[\mathrm{CV}(\%)]$ in parenthesis. Inter-animal variability (IAV) is also expressed as $\mathrm{CV}(\%)$. CL/F = apparent total plasma clearance; $\mathrm{F}$, relative bioavailability; $\mathrm{K}_{\mathrm{A}}=$ first order rate constant of absorption; $\mathrm{Q} / \mathrm{F}=$ apparent distribution clearance between the central and peripheral compartments; $V / F, V_{T} / F=$ apparent volumes of distribution of the central and peripheral compartments respectively. *, arbritary value, not estimated; ${ }^{a}$ parameter modeled as Vx (WGT/187) for (Vettorazzi et al., 2009) and $V \times(W G T / 195.6)$ in this study. 